\title{
BOUNDS FOR THE ARITHMETIC MEAN IN TERMS OF THE TOADER MEAN AND OTHER BIVARIATE MEANS
}

\author{
YUN HUA
}

Received 29 September, 2015

\begin{abstract}
We find the greatest values $\alpha_{1}$ and $\alpha_{2}$, and the least values $\beta_{1}$ and $\beta_{2}$, such that the double inequalities $\alpha_{1} T(a, b)+\left(1-\alpha_{1}\right) H(a, b)<A(a, b)<\beta_{1} T(a, b)+\left(1-\beta_{1}\right) H(a, b)$ and $\alpha_{2} T(a, b)+\left(1-\alpha_{2}\right) \bar{H}(a, b)<A(a, b)<\beta_{2} T(a, b)+\left(1-\beta_{2}\right) \bar{H}(a, b)$ hold for all $a, b>$ 0 with $a \neq b$. Here, $\bar{H}(a, b)=\sqrt{2} a b / \sqrt{a^{2}+b^{2}}, H(a, b)=2 a b /(a+b), A(a, b)=(a+$ $b) / 2$, and $T(a, b)=\frac{2}{\pi} \int_{0}^{\pi / 2} \sqrt{a^{2} \cos ^{2} \theta+b^{2} \sin ^{2} \theta} d \theta$ denote the harmonic root-square, harmonic, arithmetic and Toader means of $a$ and $b$, respectively.
\end{abstract}

2010 Mathematics Subject Classification: 26D15; 26E60

Keywords: harmonic root-square mean, harmonic, arithmetic, Toader mean, complete elliptic integrals

\section{INTRODUCTION}

In [16], Toader introduced the Toader mean $T(a, b)$ of two positive numbers $a$ and $b$ as follows:

$$
\begin{aligned}
T(a, b) & =\frac{2}{\pi} \int_{0}^{\pi / 2} \sqrt{a^{2} \cos ^{2} \theta+b^{2} \sin ^{2} \theta} d \theta \\
& = \begin{cases}2 a \mathcal{E}\left(\sqrt{1-(b / a)^{2}}\right) / \pi, & a>b, \\
2 b \mathcal{E}\left(\sqrt{1-(a / b)^{2}}\right) / \pi, & a<b, \\
a, & a=b .\end{cases}
\end{aligned}
$$

where $\mathcal{E}=\mathcal{E}(r)=\int_{0}^{\pi / 2}\left(1-r^{2} \sin ^{2} \theta\right)^{1 / 2} d \theta(r \in[0,1])$ is the complete elliptic integral of the second kind.

Recently, the Toader mean has been the object of intensive research. In particular, many remarkable inequalities for the Toader mean $T(a, b)$ can be found in the literatures [9-12, 14-16]

The author was partially supported by the Science Foundation of WeiHai Vocational college under grant No. 2016ky001. 
Let $\bar{H}(a, b)=\sqrt{2} a b / \sqrt{a^{2}+b^{2}}, H(a, b)=2 a b /(a+b), G(a, b)=\sqrt{a b}$, $A(a, b)=(a+b) / 2, S(a, b)=\sqrt{\left(a^{2}+b^{2}\right) / 2}, C(a, b)=\left(a^{2}+b^{2}\right) /(a+b)$ be the harmonic root-squre, harmonic, geometric, arithmetic, quadratic, and contraharmonic means of $a$ and $b$, respectively. Then it is known that the inequalities

$$
\bar{H}(a, b)<H(a, b)<G(a, b)<A(a, b)<S(a, b)<C(a, b)
$$

hold for all $a, b>0$ with $a \neq b$.

For $p \in \mathbb{R}, a, b>0$ with $a \neq b$ the power mean $M_{p}(a, b)$ is defined by

$$
M_{p}(a, b)= \begin{cases}\left(\frac{a^{p}+a^{p}}{2}\right)^{1 / p}, & p \neq 0, \\ \sqrt{a b}, & p=0 .\end{cases}
$$

It is well known that the power mean $M_{p}(a, b)$ is continuous and strictly increasing with respect to $p$. Many means are special case of $M_{p}(a, b)$, for example, $M_{-1}(a, b)=H(a, b)=2 a b /(a+b), M_{0}(a, b)=G(a, b)=\sqrt{a b}, M_{1}(a, b)=$ $A(a, b)=(a+b) / 2, M_{2}(a, b)=S(a, b)=\sqrt{\left(a^{2}+b^{2}\right) / 2}$.

Vuorinen [17] conjectured that

$$
M_{3 / 2}(a, b)<T(a, b)
$$

for all $a, b>0$ with $a \neq b$. This conjecture was solved by Qiu and Shen[14], and Barnard, Pearce and Richards in [6], respectively.

In [1], Alzer and Qiu presented that

$$
T(a, b)<M_{\log 2 / \log (\pi / 2)}(a, b)
$$

for all $a, b>0$ with $a \neq b$, which gives a best possible upper bound for Toader mean in terms of the power mean.

From (1.4) and (1.5) one concludes that

$$
A(a, b)<T(a, b)<S(a, b)
$$

for all $a, b>0$ with $a \neq b$.

In [11], the authors demonstrated that the double inequality

$$
\alpha S(a, b)+(1-\alpha) A(a, b)<T(a, b)<\beta S(a, b)+(1-\beta) A(a, b)
$$

holds for all $a, b>0$ with $a \neq b$ if and only if $\alpha \leq \frac{1}{2}$ and $\beta \geq \frac{4-\pi}{(\sqrt{2}-1) \pi}$.

In [15], the authors proved that the double inequalities

$$
\begin{gathered}
\alpha_{1} C(a, b)+\left(1-\alpha_{1}\right) A(a, b)<T(a, b)<\beta_{1} C(a, b)+\left(1-\beta_{1}\right) A(a, b), \\
\frac{\alpha_{2}}{A(a, b)}+\frac{1-\alpha_{2}}{C(a, b)}<\frac{1}{T(a, b)}<\frac{\beta_{2}}{A(a, b)}+\frac{1-\beta_{2}}{C(a, b)}
\end{gathered}
$$

hold for all $a, b>0$ with $a \neq b$ if and only if $\alpha_{1} \leq 1 / 4, \beta_{1} \geq 4 / \pi-1$ and $\alpha_{2} \leq$ $\pi / 2-1, \beta_{2} \geq 3 / 4$. 
The main purpose of this paper is to find the greatest values $\alpha_{1}, \alpha_{2}$ and the least values $\beta_{1}, \beta_{2}$ in $(0,1)$ such that the double inequalities

$$
\begin{aligned}
& \alpha_{1} T(a, b)+\left(1-\alpha_{1}\right) H(a, b)<A(a, b)<\beta_{1} T(a, b)+\left(1-\beta_{1}\right) H(a, b), \\
& \alpha_{2} T(a, b)+\left(1-\alpha_{2}\right) \bar{H}(a, b)<A(a, b)<\beta_{2} T(a, b)+\left(1-\beta_{2}\right) \bar{H}(a, b),
\end{aligned}
$$

hold for all $a, b>0$ with $a \neq b$.

As an application, we get a new lower bound for the complete elliptic integral of the second kind in terms of elementary functions, which improves some well-known results.

\section{PRELIMinaries AND Lemmas}

In order to establish our main result we need several lemmas, which we present in this section.

For $0<r<1$ and $r^{\prime}=\sqrt{1-r^{2}}$ Legendre's complete elliptic integrals of the first and second kinds $[7,8]$ are defined by

$$
\left\{\begin{array}{l}
\mathcal{K}=\mathcal{K}(r)=\int_{0}^{\pi / 2}\left(1-r^{2} \sin ^{2} \theta\right)^{-1 / 2} d \theta, \\
\mathcal{K}^{\prime}=\mathcal{K}^{\prime}(r)=\mathcal{K}\left(r^{\prime}\right), \\
\mathcal{K}(0)=\pi / 2, \quad \mathcal{K}(1)=\infty
\end{array}\right.
$$

and

$$
\left\{\begin{array}{l}
\mathcal{E}=\mathcal{E}(r)=\int_{0}^{\pi / 2}\left(1-r^{2} \sin ^{2} \theta\right)^{1 / 2} d \theta, \\
\mathcal{E}^{\prime}=\mathcal{E}^{\prime}(r)=\mathscr{E}\left(r^{\prime}\right) \\
\mathcal{E}(0)=\pi / 2, \quad \mathcal{E}(1)=1
\end{array}\right.
$$

respectively.

For $0<r<1$, the following formulas were presented in [4, Appendix E, pp. 474475]:

$$
\begin{array}{cc}
\frac{d \mathcal{K}}{d r}=\frac{\mathcal{E}-r^{\prime 2} \mathcal{K}}{r r^{\prime 2}}, & \frac{d \mathcal{E}}{d r}=\frac{\mathcal{E}-\mathcal{K}}{r}, \\
\frac{d\left(\mathcal{E}-r^{\prime 2} \mathcal{K}\right)}{d r}=r \mathcal{K}, & \frac{d(\mathcal{K}-\mathcal{E})}{d r}=\frac{r \mathscr{E}}{r^{\prime 2}}, \\
\mathcal{E}\left(\frac{2 \sqrt{r}}{1+r}\right)=\frac{2 \mathcal{E}-r^{\prime 2} \mathcal{K}}{1+r} .
\end{array}
$$

The following lemma can be found in [4, Theorem 3.21 (1)].

Lemma 1. $\left(\mathcal{E}-r^{\prime 2} \mathcal{K}\right) / r^{2}$ is strictly increasing from $(0,1)$ onto $(\pi / 4,1)$. 
Lemma 2 (Theorem 1.25 in [4]). For $-\infty<a<b<\infty$, let $f, g:[a, b] \rightarrow \mathbb{R}$ be continuous on $[a, b]$, and be differentiable on $(a, b)$, let $g^{\prime}(x) \neq 0$ on $(a, b)$. If $f^{\prime}(x) / g^{\prime}(x)$ is increasing (decreasing) on $(a, b)$, then so are

$$
\frac{f(x)-f(a)}{g(x)-g(a)} \text { and } \frac{f(x)-f(b)}{g(x)-g(b)} \text {. }
$$

If $f^{\prime}(x) / g^{\prime}(x)$ is strictly monotone, then the monotonicity in the conclusion is also strict.

\section{MAIN RESULTS}

Now we are in a position to state and prove our main results.

Theorem 1. The double inequality

$$
\alpha_{1} T(a, b)+\left(1-\alpha_{1}\right) H(a, b)<A(a, b)<\beta_{1} T(a, b)+\left(1-\beta_{1}\right) H(a, b)
$$

holds for all $a, b>0$ with $a \neq b$ if and only if $\alpha_{1} \leq \frac{\pi}{4}$ and $\beta_{1} \geq \frac{4}{5}$.

Proof. Without loss of generality, we assume that $a>b$. Let $t=b / a \in(0,1)$ and $r=\frac{1-t}{1+t}$, then

$$
\begin{aligned}
\frac{A(a, b)-H(a, b)}{T(a, b)-H(a, b)} & =\frac{\frac{1+t}{2}-\frac{2 t}{1+t}}{\frac{2}{\pi} \mathcal{E}^{\prime}(t)-\frac{2 t}{1+t}} \\
& =\frac{\frac{1}{1+r}-(1-r)}{\frac{2}{\pi} \mathscr{E}\left(\frac{2 \sqrt{r}}{1+r}\right)-(1-r)} \\
& =\frac{r^{2}}{\frac{2}{\pi}\left(2 \mathscr{E}-r^{\prime 2} \mathcal{K}\right)-1+r^{2}} .
\end{aligned}
$$

Let $f_{1}(r)=r^{2}, f_{2}(r)=\frac{2}{\pi}\left(2 \varepsilon-r^{\prime 2} \mathcal{K}\right)-1+r^{2}$, and

$$
f(r)=\frac{f_{1}(r)}{f_{2}(r)}=\frac{r^{2}}{\frac{2}{\pi}\left(2 \mathcal{E}-r^{\prime 2} \mathcal{K}\right)-1+r^{2}} .
$$

Then simple computations lead to

$$
\begin{aligned}
f_{1}(0) & =f_{2}(0)=0, \\
f_{1}^{\prime}(r) & =2 r \\
f_{2}^{\prime}(r) & =\frac{2}{\pi} \frac{\mathcal{E}-r^{\prime 2} \mathcal{K}}{r}+2 r \\
\frac{f_{1}^{\prime}(r)}{f_{2}^{\prime}(r)} & =\frac{1}{\frac{1}{\pi} \frac{\varepsilon-r^{\prime 2} \mathcal{K}}{r^{2}}+1} .
\end{aligned}
$$


It follows from (3.7) with Lemma 1 and Lemma 2 that $f(r)$ is strictly decreasing in $(0,1)$. Moreover, using L'Hôpital's rule we get

$$
\lim _{x \rightarrow 0^{+}} f(x)=\frac{4}{5}
$$

and

$$
\lim _{x \rightarrow 1^{-}} f(x)=\frac{\pi}{4} .
$$

Therefore, Theorem 1 follows from (3.8) and (3.9) together with the monotonicity of $f(r)$.

From Theorem 1 we get a new bound for the complete elliptic integral $\&(r)$ of the second kind in terms of elementary functions as follows.

Corollary 1. For $r \in(0,1)$ and $r^{\prime}=\sqrt{1-r^{2}}$, we have

$$
\frac{\pi}{2}\left[\frac{5}{8}\left(1+r^{\prime}\right)-\frac{r^{\prime}}{2\left(1+r^{\prime}\right)}\right]<\mathcal{E}(r)<\frac{\pi}{2}\left[\frac{2\left(1+r^{\prime}\right)}{\pi}-\left(1-\frac{4}{\pi}\right) \frac{2 r^{\prime}}{1+r^{\prime}}\right] .
$$

Proof. Without loss of generality, assume that $a>b$. Substituting $r^{\prime}=\frac{b}{a}, \alpha_{1}=$ $\frac{\pi}{4}, \beta_{1}=\frac{4}{5}$ into Theorem 1 produces Corollary 1 .

Theorem 2. The double inequality

$$
\alpha_{2} T(a, b)+\left(1-\alpha_{2}\right) \bar{H}(a, b)<A(a, b)<\beta_{2} T(a, b)+\left(1-\beta_{2}\right) \bar{H}(a, b),
$$

holds for all $a, b>0$ with $a \neq b$ if and only if $\alpha_{2} \leq \frac{\pi}{4}$ and $\beta_{2} \geq \frac{6}{7}$.

Proof. Without loss of generality, we assume that $a>b$. Let $t=b / a \in(0,1)$ and $r=\frac{1-t}{1+t}$, then

$$
\begin{aligned}
\frac{A(a, b)-\bar{H}(a, b)}{T(a, b)-\bar{H}(a, b)} & =\frac{\frac{1+t}{2}-\frac{\sqrt{2} t}{\sqrt{1+t^{2}}}}{\frac{\pi}{2} \mathcal{E}^{\prime}(t)-\frac{\sqrt{2} t}{\sqrt{1+t^{2}}}} \\
& =\frac{\frac{1}{1+r}-\frac{1-r}{\sqrt{1+r^{2}}}}{\frac{2}{\pi} \mathcal{E}\left(\frac{2 \sqrt{r}}{1+r}\right)-\frac{1-r}{\sqrt{1+r^{2}}}} \\
& =\frac{1-\frac{1-r^{2}}{\sqrt{1+r^{2}}}}{\frac{2}{\pi}\left(2 \mathcal{E}-r^{\prime 2} \mathcal{K}\right)-\frac{1-r^{2}}{\sqrt{1+r^{2}}}}
\end{aligned}
$$

Let $g_{1}(r)=1-\frac{1-r^{2}}{\sqrt{1+r^{2}}}, g_{2}(r)=\frac{2}{\pi}\left(2 \mathcal{E}-r^{\prime 2} \mathcal{K}\right)-\frac{1-r^{2}}{\sqrt{1+r^{2}}}$ and

$$
g(r)=\frac{g_{1}(r)}{g_{2}(r)}=\frac{1-\frac{1-r^{2}}{\sqrt{1+r^{2}}}}{\frac{2}{\pi}\left(2 \mathcal{E}-r^{\prime 2} \mathcal{K}\right)-\frac{1-r^{2}}{\sqrt{1+r^{2}}}} .
$$


Simple calculations show that

$$
\begin{aligned}
& g_{1}(0)=g_{2}(0)=0, \\
& g_{1}^{\prime}(r)=\frac{r\left(r^{2}+3\right)}{\left(1+r^{2}\right)^{3 / 2}}, \\
& g_{2}^{\prime}(r)=\frac{2}{\pi} \frac{\mathcal{E}-r^{\prime 2} \mathcal{K}}{r}+\frac{r\left(r^{2}+3\right)}{\left(1+r^{2}\right)^{3 / 2}}, \\
& \frac{g_{1}^{\prime}(r)}{g_{2}^{\prime}(r)}=\frac{\frac{\left(r^{2}+3\right)}{\left(1+r^{2}\right)^{3 / 2}}}{\frac{2}{\pi} \frac{\varepsilon-r^{\prime 2} \mathcal{K}}{r^{2}}+\frac{\left(r^{2}+3\right)}{\left(1+r^{2}\right)^{3 / 2}}}=\frac{1}{\frac{\frac{2}{\pi} \frac{\varepsilon-r^{\prime 2} \mathcal{K}}{r^{2}}}{\frac{\left(r^{2}+3\right)}{\left(1+r^{2}\right)^{3 / 2}}}+1} .
\end{aligned}
$$

It is easy to verify that the function $\frac{\left(r^{2}+3\right)}{\left(1+r^{2}\right)^{3 / 2}}$ is positive and strictly decreasing in $(0,1)$, then (3.17) and Lemma 1 lead to the conclusion that $\frac{g_{1}^{\prime}(r)}{g_{2}^{\prime}(r)}$ is strictly decreasing in $(0,1)$. Hence, $g(r)$ is strictly decreasing directly from Lemma 2. Moreover, the usage of L'Hôpital's rule and standard argument show that

$$
\lim _{x \rightarrow 0^{+}} g(x)=\frac{6}{7},
$$

and

$$
\lim _{x \rightarrow 1^{-}} g(x)=\frac{\pi}{4} .
$$

Thus, Theorem 2 follows from (3.18) and (3.19) together with the monotonicity of $g(r)$.

\section{COMPARISON WITH SOME WELL-KNOWN RESULTS}

Recently, the complete elliptic integrals have attracted the attention of numerous mathematicians. In particular, many remarkable properties and inequalities for the complete elliptic integrals can be found in the literature [1-3,5,11,13,18-21].

In [11], the authors obtained that

$$
\frac{\pi}{2}\left[\frac{1}{2} \sqrt{\frac{1+r^{\prime 2}}{2}}+\frac{1+r^{\prime}}{4}\right]<\mathcal{E}(r)<\frac{\pi}{2}\left[\left(\frac{4-\pi}{(\sqrt{2}-1) \pi} \sqrt{\frac{1+r^{\prime 2}}{2}}+\frac{(\sqrt{2} \pi-4)\left(1+r^{\prime}\right)}{2(\sqrt{2}-1) \pi}\right],\right.
$$

for all $r \in(0,1)$ and $r^{\prime}=\sqrt{1-r^{2}}$.

Guo and Qi [13] proved that

$$
\frac{\pi}{2}-\frac{1}{2} \log \frac{(1+r)^{1-r}}{(1-r)^{1+r}}<\mathcal{E}(r)<\frac{\pi-1}{2}+\frac{1-r^{2}}{4 r} \log \frac{1+r}{1-r},
$$

for all $r \in(0,1)$. 
It was pointed out in [11] that the bounds in (4.1) for $\mathcal{E}(r)$ are better than that in (4.2) for some $r \in(0,1)$.

Let $g(x)=\frac{5}{8}(1+x)-\frac{x}{2(1+x)}-\left[\frac{1}{2} \sqrt{\frac{1+x^{2}}{2}}+\frac{1+x}{4}\right], x \in(0,1)$. Then simple computations lead to

$$
g(x)=\frac{3 x^{2}+2 x+3-2 \sqrt{2\left(1+x^{2}\right)}(1+x)}{8(1+x)} .
$$

Since

$$
\left(3 x^{2}+2 x+3\right)^{2}-\left(2 \sqrt{2\left(1+x^{2}\right)}(1+x)\right)^{2}=(1-x)^{4}>0,
$$

we clearly see that $g(x)>0$, which shows that the lower bounds in (3.10) for $\mathcal{E}(r)$ is better than the one in (4.1).

Very recently, Yin and Qi obtained in [20] that

$$
\frac{\pi}{2} \frac{\sqrt{6+2 \sqrt{1-r^{2}}-3 r^{2}}}{2 \sqrt{2}} \leq \mathcal{E}(r) \leq \frac{\pi}{2} \frac{\sqrt{10-2 \sqrt{1-r^{2}}-5 r^{2}}}{2 \sqrt{2}} .
$$

Since

is equivalent to

$$
\frac{5}{8}(1+x)-\frac{x}{2(1+x)}>\frac{\sqrt{6+2 x-3\left(1-x^{2}\right)}}{2 \sqrt{2}}
$$

$$
\left(5 x^{2}+6 x+5\right)^{2}>8(x+1)^{2}\left(3 x^{2}+2 x+3\right)
$$

and $(x-1)^{4}>0$, the lower bound in (3.10) for $\mathcal{E}(r)$ is better than the one in (4.4).

\section{REFERENCES}

[1] H. Alzer and S.-L. Qiu, "Monotonicity theorems and inequalities for the complete elliptic integrals," J. Comput. Appl. Math., vol. 172, no. 2, pp. 289-312, 2004, doi: 10.1016/j.cam.2004.02.009.

[2] G. D. Anderson, S.-L. Qiu, M. K. Vamanamurthy, and M. Vuorinen, "Generalized elliptic integrals and modular equations," Pacific J. Math., vol. 192, no. 1, pp. 1-37, 2000, doi: 10.2140/pjm.2000.192.1.

[3] G. D. Anderson, M. K. Vamanamurthy, and M. Vuorinen, "Functional inequalities for hypergeometric functions and complete elliptic integrals," SIAM J. Math. Anal., vol. 23, no. 2, pp. 512-524, 1992, doi: 10.1137/0523025.

[4] G. D. Anderson, M. K. Vamanamurthy, and M. K. Vuorinen, Conformal invariants, inequalities, and quasiconformal maps, ser. Canadian Mathematical Society Series of Monographs and Advanced Texts. John Wiley \& Sons, Inc., New York, 1997, with 1 IBM-PC floppy disk (3.5 inch; HD), A Wiley-Interscience Publication.

[5] S. András and Á. Baricz, "Bounds for complete elliptic integrals of the first kind," Expo. Math., vol. 28, no. 4, pp. 357-364, 2010, doi: 10.1016/j.exmath.2009.12.005.

[6] R. W. Barnard, K. Pearce, and K. C. Richards, "An inequality involving the generalized hypergeometric function and the arc length of an ellipse," SIAM J. Math. Anal., vol. 31, no. 3, pp. 693-699 (electronic), 2000, doi: 10.1137/S0036141098341575. 
[7] F. Bowman, Introduction to elliptic functions with applications. Dover Publications, Inc., New York, 1961.

[8] P. F. Byrd and M. D. Friedman, Handbook of elliptic integrals for engineers and scientists, ser. Die Grundlehren der mathematischen Wissenschaften, Band 67. Springer-Verlag, New YorkHeidelberg, 1971, second edition, revised.

[9] Y.-M. Chu and M.-K. Wang, "Inequalities between arithmetic-geometric, Gini, and Toader means," Abstr. Appl. Anal., pp. Art. ID 830 585, 11, 2012, doi: 10.1155/2012/830585.

[10] Y.-M. Chu and M.-K. Wang, "Optimal Lehmer mean bounds for the Toader mean," Results Math., vol. 61, no. 3-4, pp. 223-229, 2012, doi: 10.1007/s00025-010-0090-9.

[11] Y.-M. Chu, M.-K. Wang, and S.-L. Qiu, "Optimal combinations bounds of root-square and arithmetic means for Toader mean,” Proc. Indian Acad. Sci. Math. Sci., vol. 122, no. 1, pp. 41-51, 2012, doi: 10.1007/s12044-012-0062-y.

[12] Y.-M. Chu, M.-K. Wang, S.-L. Qiu, and Y.-F. Qiu, "Sharp generalized Seiffert mean bounds for Toader mean,” Abstr. Appl. Anal., pp. Art. ID 605 259, 8, 2011, doi: 10.1155/2011/605259.

[13] B.-N. Guo and F. Qi, "Some bounds for the complete elliptic integrals of the first and second kinds," Math. Inequal. Appl., vol. 14, no. 2, pp. 323-334, 2011, doi: 10.7153/mia-14-26.

[14] S. L. Qiu and J. M. Shen, "On two problems concerning means," Journal of Hangzhou Institute of Electronic Engineering, vol. 17, no. 3, pp. 1-6, 1997.

[15] Y.-Q. Song, W.-D. Jiang, Y.-M. Chu, and D.-D. Yan, "Optimal bounds for Toader mean in terms of arithmetic and contraharmonic means," J. Math. Inequal., vol. 7, no. 4, pp. 751-757, 2013, doi: 10.7153/jmi-07-68.

[16] G. Toader, "Some mean values related to the arithmetic-geometric mean," J. Math. Anal. Appl., vol. 218, no. 2, pp. 358-368, 1998, doi: 10.1006/jmaa.1997.5766.

[17] M. Vuorinen, "Hypergeometric functions in geometric function theory," in Special functions and differential equations (Madras, 1997). Allied Publ., New Delhi, 1998, pp. 119-126.

[18] M.-K. Wang, Y.-M. Chu, S.-L. Qiu, and Y.-P. Jiang, "Convexity of the complete elliptic integrals of the first kind with respect to Hölder means," J. Math. Anal. Appl., vol. 388, no. 2, pp. 1141-1146, 2012, doi: 10.1016/j.jmaa.2011.10.063.

[19] M.-K. Wang, Y.-M. Chu, Y.-F. Qiu, and S.-L. Qiu, "An optimal power mean inequality for the complete elliptic integrals," Appl. Math. Lett., vol. 24, no. 6, pp. 887-890, 2011, doi: 10.1016/j.aml.2010.12.044.

[20] L. Yin and F. Qi, "Some inequalities for complete elliptic integrals," Appl. Math. E-Notes, vol. 14, pp. 193-199, 2014.

[21] L. Zhou, S. Qiu, and F. Wang, "Inequalities for the generalized elliptic integrals with respect to Hölder means," J. Math. Anal. Appl., vol. 386, no. 2, pp. 641-646, 2012, doi: 10.1016/j.jmaa.2011.08.026.

\section{Author's address}

\section{Yun Hua}

Weihai Vocational College, Department of Information Engineering, Weihai City 264210, ShanDong province, P. R. CHINA.

E-mail address: xxgcxhy@163.com 